\title{
Voix de faits : vers une « dénarrativisation » du fait divers chez François Bon et Laurent Mauvignier
}

Frédéric Martin-Achard

\section{OpenEdition}

\section{Journals}

Édition électronique

URL : http://journals.openedition.org/recherchestravaux/965

ISSN : 1969-6434

Éditeur

UGA Éditions/Université Grenoble Alpes

Édition imprimée

ISBN : 978-2-37747-056-3

ISSN : 0151-1874

\section{Référence électronique}

Frédéric Martin-Achard, « Voix de faits : vers une « dénarrativisation » du fait divers chez François Bon et Laurent Mauvignier », Recherches \& Travaux [En ligne], 92 | 2018, mis en ligne le , consulté le 08 septembre 2020. URL : http://journals.openedition.org/recherchestravaux/965

Ce document a été généré automatiquement le 8 septembre 2020

(c) Recherches \& Travaux 


\title{
Voix de faits : vers une
} « dénarrativisation » du fait divers chez François Bon et Laurent Mauvignier

\author{
Frédéric Martin-Achard
}

Parmi les nombreuses fictions narratives d'expression française qui s'inspirent ou s'emparent d'un fait divers, les deux textes sur lesquels portera cette étude, Un fait divers (1990) de François Bon et Ce que j'appelle oubli (2011) de Laurent Mauvignier ${ }^{1}$, ont la particularité de reléguer immédiatement la référence au fait divers à leur seuil, en quatrième de couverture: chez François Bon, un article du Courrier de l'Ouest qui annonce la tenue du procès en assise et reprend le récit du crime y est reproduit, tandis que chez Mauvignier figure la mention suivante en italique : "Cette fiction est librement inspirée d'un fait divers, survenu à Lyon, en décembre 2009. » Le titre du roman de Bon est ambivalent : s'il souligne d'emblée l'étroitesse du rapport entre le fait divers et l'œuvre romanesque, il permet, d'une part, de circonscrire là encore le fait divers au péritexte du roman et, d'autre part, d'affirmer, par l'usage de l'article indéfini, le caractère indifférent ou la valeur générique du fait en question. C'est ce rapport entretenu par le récit à la matière du fait divers que nous allons interroger. La seconde particularité commune aux récits de Laurent Mauvignier et au roman de François Bon est qu'au récit journalistique de l'événement va se substituer un dispositif énonciatif et narratif singulier, dans lequel la voix occupe une place centrale. Dans un ouvrage consacré à l'écriture du fait divers dans la presse française au xix siècle, Laetitia Gonon montre que ces récits, souvent très brefs, reprennent des modèles textuels volontiers stéréotypés et présentent dès lors différents types de figement: narratif, "fondé sur le retour de propositions identiques, de titres et d'amorces", syntaxique, qui « repose alors sur des expressions toutes faites intervenant de façon rituelle » et, enfin, « de l'ordre chronologique ${ }^{2}$ ». L'hypothèse que nous formulerons est que les dispositifs de François Bon et Laurent Mauvignier ont une incidence sur ces différentes formes de figement par l'exhibition des voix qui constituent le fait divers, la 
déconstruction de la chronologie du récit, la monstration enfin et la critique des expressions figées qui circulent sur le fait divers. Le corollaire de cette hypothèse est que ces phénomènes concourent à une forme de «dénarrativisation » du fait divers et d'affaiblissement de sa valeur événementielle.

\section{Exposition des voix}

2 Contrairement au récit du fait-diversier dans lequel «le journaliste est un narrateur essentiellement effacé ${ }^{3}$ ", celui de Laurent Mauvignier se présente comme le soliloque ${ }^{4}$ d'un je qui s'adresse à un allocutaire muet, lequel n'est autre que le frère d'un jeune homme battu à mort par quatre vigiles pour avoir bu une canette de bière dans un supermarché sans l'avoir achetée au préalable. Cette voix narrative à l'identité incertaine s'exhibe, met en scène ses questionnements, ses doutes, ses détours et ses revirements :

[I]l marche avec les mouvements et les écarts qu'il faut pour éviter les Caddies et les gens - mais je ne sais pas s'il va tout de suite vers les bières, je ne crois pas, il tombe dessus presque par hasard, très vite, à droite dans l'entrée du magasin et non pas au fond à gauche comme il croit s'en souvenir (C, p. 11).

L'usage très fréquent du tiret marque ici le décrochage énonciatif du commentaire, de la modalisation épistémique, mais il permet souvent de faire entendre plusieurs voix, de déployer plusieurs strates énonciatives simultanées. Non seulement le locuteur modalise son récit (il affirme une forme de non-savoir, puis récuse comme peu probable les faits envisagés avant de proposer une autre hypothèse), mais il le " fictionnalise » en attribuant des états mentaux à un personnage, et cette fois-ci de façon assertive $e^{5}$. Aussi le récit opère-t-il d'emblée un premier pas de côté en opérant une « fictionnalisation » du fait divers.

Mais la modalisation peut aussi être autonymique ${ }^{6}$ et porter sur les mots employés par un locuteur précautionneux, associée à diverses formes d'épanorthoses :

[C]e qu'il a fait, non pas tenter de nier lorsqu'il les a vus arriver sur lui et qu'ils se sont, je ne dirais pas abattus sur lui, parce qu'ils étaient lents et calmes et qu'ils n'ont pas du tout fondu comme l'auraient fait, disons, des oiseaux de proie, non, pas du tout, au contraire ils se sont arrêtés devant lui (C, p. 8).

5 Le discours mêle les marques de reformulation du propos et des boucles réflexives sous forme d'incidente ou d'incise. Les deux gloses relèvent de deux formes distinctes de non-coïncidence du dire: entre les mots et les choses dans le premier cas et interlocutive entre les co-énonciateurs (en l'occurrence le je et son allocutaire) dans le second cas. Le discours progresse donc en exhibant son dire par interruptions ou suspensions (l'ellipse du participe passé), corrections et rectifications, commentaires et revirements à la limite de la palinodie. Les hésitations, reformulations et précautions lexicales du locuteur tendent à la fois vers ce que Jacqueline Authier-Revuz décrit comme une "nomination au terme d'une recherche", qui creuse dans le dire "l'écart de la difficulté à nommer dont ce dire marque, sinon la solution, au moins le terme », et vers la "nomination avec remplacement", parcours "accidenté, heurté, d'un dire effectué puis remplacé par un autre dire effaçant, annulant le premier ${ }^{7}$ ». Dans les deux cas, les événements se trouvent dilatés et leur narration étirée par l'exposition du travail de nomination. 
6 Si le récit est fondamentalement monologal ${ }^{8}$, il n'est pas pour autant monologique, c'est-à-dire que la voix narrative est sans cesse traversée par des discours hétérogènes qu'elle représente sous différents modes, et ce dès les premières lignes de l'incipit :

[E]t ce que le procureur a dit, c'est qu'un homme ne doit pas mourir pour si peu, qu'il est injuste de mourir à cause d'une canette de bière que le type aura gardée assez longtemps entre les mains pour que les vigiles puissent l'accuser de vol et se vanter, après, de l'avoir repéré et choisi parmi les autres, là, qui font leurs courses (C, p. 8)

7 La proposition semi-clivée met en relief les propos du procureur au discours indirect par lequel s'ouvre donc le récit; d'emblée, d'autres voix que celle du locuteur s'imbriquent les unes dans les autres : le discours des vigiles est narrativisé ( l'accuser de vol») puis représenté au style indirect («se vanter [...] de l'avoir repéré...»). Ces voix que le locuteur donne à entendre sont celles de la victime, du procureur, mais aussi de la police, des journalistes, des collègues, du patron et de la femme du frère de la victime et enfin des clients de la boucherie tenue par les parents. Les discours hétérogènes sont représentés dans de multiples formes, parfois mixtes, des plus indirectes aux plus directes :

[E]t il a le temps de se dire que l'autre, avec la barbe pas plus épaisse qu'un trait qui dessine la mâchoire, celui-là ne doit pas aimer courir, il est lourd, plus que lui, je peux courir plus vite se dit-il (C, p. 16).

On passe ici d'un discours indirect à un discours direct avec une incise de discours intérieur, mais sans l'appareil typographique du discours rapporté. La fluidité du glissement est assurée aussi bien par l'absence de signes typographiques que par une émancipation progressive du discours indirect vers une forme intermédiaire d'indirect libre au présent («il est lourd»), dès lors que le discours rapporté se détache de la proposition conjonctive ${ }^{9}$. Le dialogisme se manifeste donc par un entrelacement de discours rapportés dans une grande labilité de formes, favorisée par l'absence de signes de ponctuation spécifiques; le lissage des formes provoque une relative indistinction entre les voix, un amuïssement de leurs traits distinctifs. Pour Laetitia Gonon, le fait divers journalistique est un réseau d'interdiscours, tissé d'emprunts à diverses sources, notamment policière et médicale, dont la circulation et l'enchevêtrement rendent l'identification parfois impossible ${ }^{10}$; «de plus, les faits-diversiers tendent à imiter ces discours officiels, en recourant à des collocations ou des formes syntaxiques figées qui donnent l'impression au lecteur que la police, la justice, l'expertise médicale, ont été convoquées ${ }^{11}$. » Chez Laurent Mauvignier, l'hétérogénéité demeure marquée ; au fondu $\mathrm{du}$ fait divers, le récit substitue des formes visibles de dialogisation de la voix narrative.

9 Chez François Bon, le dispositif expose cette multiplicité des énonciateurs, dissimulée par le récit de faits divers, dans un roman polyphonique, mettant en scène une pluralité de locuteurs en une suite de monologues alternés et sans l'intervention d'un narrateur. Le réseau de voix se trouve donc décomposé en une succession de monologues qui débutent, chacun, par la désignation du locuteur. Outre les homoénonciateurs (les victimes, les témoins, mais aussi le coupable) et les paraénonciateurs ${ }^{12}$ (l'inspecteur, le légiste, le substitut du procureur, le psychiatre expert), il faut ajouter toute une série de ce qu'on peut appeler des "méta-énonciateurs " (metteur en scène, auteur, comédien, directeur de la photographie), car le fait divers fait l'objet d'une adaptation cinématographique au sein du roman. Le texte se pare donc d'une dimension réflexive : il s'agit de réfléchir à la représentation filmique après coup du fait divers, tout en assurant la restitution des faits et de leurs répercussions sur 
leurs principaux acteurs trente mois plus tard. Mais, paradoxalement, si le roman de François Bon est nettement polyphonique et met tous les énonciateurs sur le même plan - contrairement à la voix narrative chez Laurent Mauvignier toujours en position surplombante de surénonciation ${ }^{13}-$ il n'est que très marginalement dialogique, ni même dialogal dans la mesure où les monologues ne se répondent pas. Dans les deux cas, il s'agit bien de montrer la pluralité des voix et des discours qui constitue le fait divers et de jouer de cette multiplicité contre le récit.

\section{Déconstruction de la chronologie}

Le dispositif d'Un fait divers, qui divise le texte en douze parties, a également des conséquences sur la chronologie narrative. Hormis la première partie, qui constitue une sorte de prologue, les onze suivantes sont intitulées "déposition » et comportent un sous-titre entre parenthèses, syntagme nominal sans déterminant qui annonce de façon plus ou moins transparente le thème principal de la déposition (rébellion, jalousies, violence...). L'ordre de ces différentes « dépositions » n'est pas strictement chronologique : les deux premières évoquent majoritairement le début de la nuit du crime, tandis que la troisième constitue une première analepse et se concentre sur la rencontre entre les personnages principaux - le meurtrier et son ex-compagne - ; les dépositions quatre à six reviennent principalement au huis clos de la nuit du crime (un monologue du meurtrier évoque son arrêt à Romorantin avant d'arriver au Mans); la septième marque une nouvelle analepse en narrant l'installation du couple à Marseille ; la huitième est un retour à la nuit du crime; les dépositions neuf et dix reprennent le prolongement de la vie marseillaise, jusqu'à la séparation du couple. Enfin, la onzième et dernière déposition rappelle le dénouement du fait divers, puis le procès, et se clôt, dans une prolepse et une ellipse temporelle, sur le monologue du meurtrier anticipant sa libération future. On pourrait, en première approximation, considérer que le temps de l'histoire est double : au récit du fait divers s'entremêle celui de la rencontre entre les deux personnages principaux jusqu'à la dégradation de leur relation - les deux histoires étant énoncées depuis un moment de trente mois postérieur au fait divers, celui du procès, dans des monologues remémoratifs, proches d'une narration intercalaire. Ainsi, l'histoire seconde (les parties en analepse narrant la rencontre) résulterait d'une volonté d'ordonnancement à la fois chronologique et causal des faits pour aboutir au dénouement, au geste criminel ${ }^{14}$.

Or, le dispositif de François Bon est plus complexe. D’une part, sur le plan de la diégèse, la progression des deux histoires en alternance n'est pas strictement chronologique. Alors que les protagonistes du fait divers se remémorent majoritairement la nuit du drame, certains monologues narrent l'hospitalisation ultérieure ou la reconstitution des faits ou encore le procès près de trois ans après; de même, les para-énonciateurs décrivent leurs interventions après le crime (l'inspecteur ou le médecin légiste) ou leur rôle lors du procès (l'avocat et l'expert psychiatre). Mais surtout, une nouvelle strate temporelle apparaît au sein de presque chaque déposition par le biais des membres de l'équipe de tournage qui prépare un film sur le fait divers. Ainsi, un comédien, le metteur en scène, le directeur de la photographie, deux comédiennes et l'auteur rapportent le travail préparatoire sur les lieux du tournage, la rencontre avec l'équipe, les conditions du tournage, reviennent sur le choix du sujet et les options de mise en scène, dans une fiction réflexive ou "métafiction » ultérieure qui vient redoubler la 
restitution du fait divers. D'autre part, le temps de l'énonciation des différents monologues est mouvant, difficilement situable. S'ils sont tous rétrospectifs, le moment de la remémoration varie: tous ne s'énoncent pas au moment du procès comme la division du texte en onze "dépositions » et comme certains déictiques le laisseraient entendre. Hormis la fiction seconde, dont la temporalité est celle du tournage, on peut constater que le monologue de l'avocat ( $F D, \mathrm{p} .143-149)$ fait référence au procès au passé composé, tout comme celui de son client qui clôt le roman (FD, p. 55-57).

Dans Ce que j'appelle oubli, le soliloque est proféré lui aussi dans un après-coup non seulement par rapport au fait divers, mais aussi par rapport au procès. Outre cette postériorité aux faits rapportés, le temps de l'énonciation est insituable précisément. Le récit du fait divers est mené au présent, mais, comme chez François Bon, l'ordre en est sensiblement perturbé par des prolepses ${ }^{15}$ et la remémoration de souvenirs ${ }^{16}$ de la victime, antérieurs au meurtre, qui confère une épaisseur temporelle au texte. Mais plus encore que ces transgressions à la chronologie du fait divers, le récit de Laurent Mauvignier remet en cause le critère définitoire principal du fait divers selon Barthes : son immanence. En effet, selon Barthes, le fait divers « est une information totale », qui " contient en soi tout son savoir »; c'est " une structure fermée ${ }^{17}$ ». Or, précisément, contre la clôture narrative, le texte de Mauvignier joue la non-clôture de la phrase puisque le texte consiste en une phrase ou plutôt en moins qu'une phrase graphique, car il débute par une minuscule et ne se termine pas par un point. L'absence de bornes initiales et finales de la phrase graphique est redoublée par des phénomènes syntaxiques et énonciatifs : le soliloque débute par la conjonction de coordination " et " en minuscule, laissant envisager l'ellipse d'une ou plusieurs propositions dans une phrase prise in medias res. De même, le récit se conclut par la rumination intérieure de la victime et s'arrête sur un ultime tiret simple ouvrant, qui, à la fois, renvoie à une nouvelle incidente élidée, hors texte (comme le début invite à considérer un hors-texte préalable), et marque le silence d'une pause musicale. Non seulement le fait divers n'est pas tout entier dans le récit, mais encore la voix qui l'interroge ne parvient pas à l'expliquer définitivement, à lui attribuer une signification dernière. À la clôture, à l'immanence du fait divers, le texte oppose l'ouverture de la quête du sens, l'interrogation toujours relancée de l'événement, de sa violence, et des mots pour le dire.

\section{Monstration et critique du figement linguistique}

13 Car il s'agit d'interroger aussi bien ce que le fait divers révèle du réel social que les mots, les expressions figées, pour le dire. Chez François Bon une opposition se dessine entre la langue commune, vulgaire, du fait divers, "à l'abord informe», et la langue littéraire, qui reste à trouver, «à l'écart des faits pour garder sa hauteur, quand les faits sont choisis justement pour n'avoir rien à signaler » (FD, p. 29-30). La critique du « déjàdit » est portée dans le roman par le comédien et l'avocat :

Il y a eu le réquisitoire des parties civiles: "soirée de haine ", ou bien "une effroyable descente aux enfers ", c'est si facile de coller les mots qui plaquent sur la réalité complexe tel registre d'une autre, plus symbolique qui lui préexiste. [...] Et " effroyable » aurait été plus efficace après « descente aux enfers ", qu'il réduit au cliché, parce que voilà seulement le cas que fait l'ordre du monde des épines qui le traversent : les faire coïncider avec les petites cases pour lesquelles des mots sont prévus (FD, p. 144-145). 
L'avocat se livre à une critique linguistique des phénomènes phraséologiques propres aux discours du fait divers, dont s'emparent les parties civiles. Le syntagme nominal $\mathrm{N}_{1}$ de $\mathrm{N}_{2}$ " soirée de haine » présente certains traits de figement tels que l'impossibilité d'y insérer un autre constituant ${ }^{18}$, mais ces deux substantifs restent libres et son sens est compositionnel. Il se rapproche de la collocation ${ }^{19}$ sans constituer pleinement un phrasème ${ }^{20}$. En revanche, le deuxième syntagme complexe, qualifié de «cliché", présente un plus important degré de figement syntaxique : les deux substantifs sont contraints, à la limite de la composition ${ }^{21}$, tandis qu' " effroyable ", sur lequel porte la critique de l'avocat, peut s'interpréter comme une épithète de nature ou relever de la collocation. Le reproche de l'avocat est aussi grammatical et porte sur l'antéposition de l'épithète, adjectif de troisième type, quand la postposition lui restituerait la force sémantique d'un adjectif qualificatif. L'ordre des mots de ce langage affadi ne bouscule pas l'ordre du monde établi.

Si les para-énonciateurs sont porteurs d'une critique de la phraséologie propre au fait divers, ils en assurent aussi la reproduction. Ce sont dans les monologues de l'inspecteur, mais aussi du substitut du procureur et de l'expert psychiatre que sont représentées la plupart des expressions figées et des unités phraséologiques qui rappellent une forme de sociolecte professionnel. Le premier trait caractéristique du monologue de l'inspecteur est l'usage récurrent de la voix passive et en particulier du passif incomplet, sans réalisation du complément d'agent ${ }^{22}$ :

Qu'en présence du premier substitut du procureur constatations furent faites rue de l'Abbé-Gruget [...] d'une Mobylette attachée par chaîne et cadenas à la grille d'un entresol et cadenas correspondant à la clé trouvée sur Frank [...]. Que témoignages furent recueillis de son passage en milieu de nuit au restaurant routier de Romorantin, comme témoignages furent recueillis de l'attente en différents points de la ville [...]. Que constatations furent faites de l'heure de son intrusion dans l'appartement de Charles Catherine et des dégradations volontaires qui y furent commises, en particulier sectionnement évident des fils du téléphone ( $F D$, p. 16).

16 Le passif incomplet, qui coïncide ici avec des constructions binaires proches de la collocation, écarte tout agent humain et rappelle ainsi l'impersonnalité du rapport de police; mais il n'est pas, pour autant, le signe d'une recherche de réalisme sociolinguistique de la part de François Bon. Les tournures passives et les collocations se répètent et forment un chiasme : le monologue ne mime ni la spontanéité de l'oral ni le figement d'un technolecte policier. Le chiasme, les répétitions et l'anaphore de la conjonction «que » en tête de phrase concourent à des effets qui relèvent plus d'une forme de préciosité rhétorique que d'une oralité spontanée.

Le second trait essentiel consiste dans les collocations et les épithètes de nature: " époux légitime ", « contusions violentes à la tête ", « engageant conversation ", « s'en rendit maitre », «traces vives ", « l'appartement sis 119 avenue René-Gasnier ${ }^{23}$ », etc. Enfin, le troisième trait caractéristique de ce style des para-énonciateurs réside dans l'absence fréquente de déterminant ou plutôt dans l'usage massif de l'article zéro, souvent interprété par les grammaires comme une marque de figement ${ }^{24}$. Chez François Bon, il intervient aussi bien dans des constructions plus ou moins figées telles que des collocations et locutions ("s'en rendit maître", «à usage chirurgical ", "après enquête »), que dans des appositions («Frank, accusé principal ») ou des structures plus inattendues ( «comme chaque année à même époque $\left.{ }^{25} »\right)$. Des études linguistiques récusent l'idée selon laquelle l'article zéro serait un indice de figement ${ }^{26}$, ce que semblent aussi montrer les exemples précités. Toutefois, ce qui importe ici, ce n'est pas 
tant le fait que l'article zéro constitue effectivement un indice linguistique de figement, mais qu'il contribue stylistiquement à générer un effet de figement, un effet de phraséologie officielle.

Dans Ce que j'appelle oubli, la voix de chaque énonciateur est peu distincte et la représentation de discours figés du fait divers moins prégnante. On peut cependant en relever quelques traces chez des énonciateurs secondaires. Il ne s'agit pas tant de phénomènes de figement syntaxique que de stéréotypes, entendus comme « un schème collectif figé ${ }^{27}$ ", ou de lieu commun, au sens non spécifique d'idée banale, doxique. Toutefois, ces lieux communs prennent une forme linguistique privilégiée, qui est celle de l'énoncé générique ${ }^{28}$ :

[E]t tes collègues te plaindront au moins le temps d'un après-midi, et même ton patron dira que c'est une honte de mourir pour si peu, on ne doit pas mourir pour ça, dans quel monde on vit dans quel monde, diront-ils [...]

[O]u les clients de votre père [...], hésitant un peu, faussement timides, au lieu de dire comme d'habitude, il fait beau aujourd'hui, prendre la mine de circonstance, on a appris pour votre fils, dans quel monde on vit, hein (C, p. 56 et 33)

Ces énoncés génériques ont tous en commun une forme d'impersonnalité (présentatif, présent gnomique, pronom " on ») qui permet leur circulation au sein du récit d'un énonciateur à un autre : les propos du procureur se retrouvent chez les collègues de travail du frère de la victime, lesquels partagent les mêmes lieux communs que les clients de la boucherie des parents. Et c'est cette circulation que la voix narrative déplore :

[L]es mots que le procureur et la police ont dit et répétés et qu'on a entendus dans les rues et les journaux, jetés sur la voie publique comme pour y faire pousser des fleurs (comme si toute la vérité du monde tenait là-dedans !) (C, p. 37-38) Le locuteur souligne l'inadéquation entre ces formules standardisées et impersonnelles, et le réel, qu'elles réduisent. Ces mots de tout le monde ne sont les mots de personne ; ils relèvent d'un " déjà-dit » et renvoient à une doxa suspecte, car accréditant l'ordre d'un monde rendant une telle violence possible.

En revanche, chez François Bon, l'effet de figement se mêle à d'autres traits stylistiques tels que des perturbations dans l'ordre des mots (accumulation de constituants à gauche de la phrase, conjonction "que » en tête de phrase) et de nombreuses phrases averbales. Ce dernier trait est particulièrement significatif : associée à de nombreux déverbaux et à une forte présence des modes non personnels du verbe, la phrase averbale procède à une forme de réification du fait divers. Le procès se fait chose; et cet affaiblissement de la valeur événementielle du fait divers s'accompagne de l'évacuation de tout agent humain par le passif incomplet. C'est que, in fine, l'événement importe peu, il est «tout juste un froissement provisoire rapporté au temps arrêté de la ville et des routes » (FD, p. 14).

En définitive, le fait divers chez François Bon et Laurent Mauvignier n'est pas tant exploité pour ses potentialités narratives, qu'interrogé pour ce qu'il recèle d'un savoir à la fois intime et collectif, relevant des sciences humaines et sociales. Les deux auteurs s'inscrivent ainsi dans un pan important du roman contemporain ${ }^{29}$, qui questionne les discours portés sur le fait divers, se penche sur ses répercussions subjectives ${ }^{30}$, refuse l'immanence de l'événement, et se méfie du romanesque trop évident qui s'en dégage ${ }^{31}$. Ce travail de sape du récit n'est pas sans rappeler, mutatis mutandis, celui que décèle Weinrich dans la réception du mythe, « réduit dans son caractère narratif et immobilisé dans son caractère événementiel ${ }^{32}$ ». Pour Weinrich, nous ne parlons plus 
«narrativement» d'un sujet sérieux, mais dans un langage argumentatif, évitant savamment toute séquence narrative. Certes, les textes de Laurent Mauvignier et François Bon ne perdent pas complètement leur caractère narratif, ni le fait divers sa force événementielle. Certes encore, le discours littéraire n'est pas explicitement argumentatif comme celui de la science désigné par Weinrich: il interroge, mais n'asserte pas. Toutefois, cette comparaison avec le mythe nous éclaire sur la place du fait divers dans ces deux récits : il est mineur et doit donc être rejeté en marge du texte ; mais il est aussi trop sérieux pour être simplement raconté et nécessite ainsi d'être interrogé, déconstruit, creusé «comme on forerait dans une épaisseur une galerie de mine » (FD, p. 129).

\section{NOTES}

1. F. Bon, Un fait divers, Paris, Minuit, 1990 (désormais FD) et L. Mauvignier, Ce que j'appelle oubli, Paris, Minuit, 2011 (désormais $C$ ).

2. L. Gonon, Le Fait divers criminel dans la presse française quotidienne du XIX siècle, Paris, Presses Sorbonne nouvelle, 2012, p. 57.

3. A. Petitjean, "Les faits divers: polyphonie énonciative et hétérogénéité textuelle ", Langue française, $\mathrm{n}^{\circ} 74,1987$, p. 75 . Sauf indication contraire, les soulignements sont de l'auteur de chaque citation.

4. Nous considérons que le soliloque romanesque se distingue du monologue intérieur par l'adresse à un allocutaire, des indices que le discours est rapporté, proféré ou écrit, et l'absence ou la présence ténue de traits stylistiques du discours intérieur. Voir F. Martin-Achard, Voix intimes, voix sociales. Usages du monologue romanesque aujourd'hui, Paris, Classiques Garnier, coll. «Étude de littérature des Xxe et XxI ${ }^{\mathrm{e}}$ siècles », 2011, p. 28-31.

5. Selon la thèse de Dorrit Cohn, pour qui « l'esprit d'un personnage imaginaire peut être connu d'une manière dont ne peut pas l'être celui d'une personne réelle ». (D. Cohn, Le Propre de la fiction [1999], Cl. Hary-Schaeffer (trad.), Paris, Seuil, coll. « Poétique », 2001, p. 181.)

6. Voir J. Authier-Revuz, Ces mots qui ne vont pas de soi. Boucles réflexives et non-coïncidences du dire, t. II, Paris, Larousse, coll. « Sciences du langage », 1995.

7. Ibid., p. 605 et 621.

8. Nous considérons comme "monologal» un discours qui ne comporte qu'un seul locuteur, tandis que «dialogal " implique l'alternance effective des locuteurs. Par "dialogisme», nous entendons les phénomènes par lesquels un énoncé, dans ses interactions avec d'autres énoncés, laisse entendre une ou plusieurs voix (ou énonciateurs) autre(s) que celle du locuteur et linguistiquement marquées. On conservera "polyphonie»dans un sens non spécifique et macrotextuel pour désigner un texte mettant en scène une pluralité de locuteurs.

9. Cela confirme l'hypothèse, défendue par Laurence Rosier, d'un continuum de l'indirect au direct par un processus d'actualisation (L. Rosier, Le Discours rapporté. Histoire, théories, pratiques, Paris/Bruxelles, Duculot, 1999, p. 158).

10. Pour M. Pêcheux, « le propre de toute formation discursive est de dissimuler [...] l'objectivité matérielle et contradictoire de l'interdiscours». (M. Pêcheux, L'Inquiétude du discours, textes choisis et présentés par D. Maldidier, Paris, Édition des Cendres, 1990, p. 227).

11. L. Gonon, ouvr. cité, p. 161. 
12. Selon la typologie d'A. Petitjean, art. cité, p. 74.

13. Voir A. Rabatel, «L'effacement énonciatif dans les discours rapportés et ses effets pragmatiques », Langages, nํ 156, « Effacement énonciatif et discours rapportés », 2004, p. 9.

14. Genette parle d'« arbitraire du récit » ou de « déterminations rétrogrades » pour désigner « la détermination des moyens par les fins, et [...], des causes par les effets.» (G. Genette, «Vraisemblance et motivation", Communications, $n^{\circ} 11,1968$, p. 18.) Voir aussi J.-M. Adam, Les Textes. Types et prototypes [1992], Paris, Armand Colin, 2008, p. 46 et L. Gonon, ouvr. cité, p. 41.

15. Voir par exemple le passage au futur : C, p. 17 et 29.

16. Voir C, p. 43 et 50 .

17. R. Barthes, "Structure du fait divers » [1964], dans Essais critiques, CEuvres complètes, t. II, É. Marty (éd.), Paris, Seuil, 2002, p. 443.

18. Sur les critères de figement des noms composés de type Nom de Nom, voir G. Gross, Les Expressions figées en français. Noms composés et autres locutions, Paris, Ophrys, 1996, p. 53-59.

19. Au sens large, " [1] es collocations sont des mots qui tendent à apparaître ensemble.» (A. Tutin et F. Grossmann, "Collocations régulières et irrégulières: esquisse de typologie du phénomène collocatif », Revue française de linguistique appliquée, VII, 2002, p. 8.)

20. Pour I. Mel'čuk, un phrasème est « un syntagme qui ne peut pas être construit selon les règles générales de la langue», par opposition au syntagme libre, non contraint et de sens compositionnel. (I. Mel'čuk, "Phrasèmes dans le dictionnaire ", dans J.-C. Anscombre \& S. Mejri (dir.), Le Figement linguistique : la parole entravée, Paris, Champion, 2011, p. 42.) Mel'čuk considère la collocation comme un phrasème semi-contraint, contraint dans l'un de ses constituants, et le cliché comme un phrasème de sens compositionnel mais syntaxiquement complètement contraint.

21. Voir G. Gross, art. cité, p. 42.

22. A. Petitjean remarque que ce type de passif abonde dans les faits divers qui réservent l'énigme pour la fin de l'article (art. cité, p. 84).

23. $F D$, respectivement, p. $21,53,61,43,53$ et 54 .

24. S'agissant des titres de fait divers, pour lesquels l'article zéro exerce pleinement sa fonction d'étiquetage, L. Gonon estime qu' « [o]n atteint, avec l'article zéro, un degré de figement et de généricité important. » (ouvr. cité, p. 65.)

25. $F D$, respectivement p. 43, 60, 53 et 120 .

26. Voir J.-C. Anscombre, "L'article zéro en français: un imparfait du substantif? ", Langue française, $\mathrm{n}^{\circ}$ 72, «Déterminants et détermination », 1986, p. 7. Pour L. Benetti, «il n'y a pas de stricte "réciprocité" entre l'absence d'article réalisé et le caractère figé de ces séquences.» (L. Benetti, L'Article zéro en français contemporain. Aspects syntaxiques et sémantiques, Bern, Peter Lang, coll. «Publications universitaires européennes », 2008, p. 15.)

27. R. Amossy \& A. Heschberg-Pierrot, Stéréotypes et clichés. Langue, discours, société, Paris, Nathan, 1997, p. 64.

28. L'énoncé générique renvoie à un ON-locuteur, entité collective proche de la doxa, qu'Anscombre définit comme «une communauté linguistique présentée comme générale, et dépassant les limites $\mathrm{du}$ locuteur et de ses éventuels allocutaires». (J.-C. Anscombre, "Stéréotypes, gnomicité et polyphonie : la voix de son maître », dans L. Perrin (dir.), Le Sens et ses voix. Dialogisme et polyphonie en discours, Metz, Université Paul Verlaine, coll. «Recherches linguistiques ", 2006, p. 365.)

29. On peut penser, entre autres, à L'Adversaire d'E. Carrère (2000), Mariage mixte de M. Weitzmann publié la même année, Les Jouets vivants de J.-Y.Cendrey (2005), Viol de D. Sallenave (1997), ou plus récemment La petite femelle (2015) et La Serpe (2017) de Ph. Jaenada.

30. E. Carrère, par exemple, affirme n'avoir réussi à écrire L'Adversaire qu' " [e]n consentant à la première personne » et en se libérant du modèle impersonnel de T. Capote (E. Carrère, «Capote, 
Romand et moi », Il est avantageux d'avoir où aller [2016], Paris, Gallimard, coll. « Folio », 2017, p. 261).

31. Voir D. Viart, «Fiction et faits divers », dans D. Viart et B. Vercier, La Littérature française au présent. Héritage, modernité, mutations, Paris, Bordas, 2008, p. 235-251.

32. H. Weinrich, "Structures narratives du mythe» [1970], Conscience linguistique et lectures littéraires, Paris, Éd. Maison des sciences de l'homme, 1989, p. 17.

\section{AUTEUR}

\section{FRÉDÉRIC MARTIN-ACHARD}

Université Jean Monnet (Saint-Étienne), CIEREC (EA 3068).

Frédéric Martin-Achard est maître de conférence en stylistique et littérature françaises des $\mathrm{Xx}^{\mathrm{e}}$ et XXI ${ }^{e}$ siècles à l'Université Jean Monnet de Saint-Étienne, membre du CIEREC (EA 3068). Il a publié Voix intimes, voix sociales. Usages du monologue romanesque aujourd'hui (Classiques Garnier, 2017) et des articles sur Bon, Mauvignier, Serena, Rolin, Yourcenar, Barthes, sur le discours intérieur et l'intériorité, le personnage romanesque et l'identité personnelle, le « récit périurbain ", ainsi qu'une édition critique de conférences inédites de Sartre. 